DOI: https://doi.org/10.11144/Javeriana.umed60-4.sgar

\title{
Infarto agudo de miocardio y bloqueo de rama izquierda: utilidad de los criterios de Sgarbossa
}

\section{Acute Myocardial Infarction and Left Branch Bundle Block: Utility of Sgarbossa Criteria}

Recepción: 27/12/2018 | Aceptación: 12 Abril 2019

\author{
Daniel Augusto Martín Arsanios ${ }^{a}$ \\ Residente de Medicina Interna de la Universidad de la \\ Sabana, Chía, Colombia \\ Elías Quintero \\ Médico de la Universidad de La Sabana, Chía, \\ Colombia \\ Santiago Benjumea HenaO \\ Médico de la Universidad de La Sabana, Chía, \\ Colombia \\ Álvaro Arteaga \\ Médico de la Universidad de La Sabana, Chía, \\ Colombia \\ VANESA RoBles \\ Médica de la Universidad de La Sabana, Chía, \\ Colombia \\ Daniel Vesga \\ Residente de Medicina Interna, Universidad Pontificia \\ Bolivariana, Medellín, Colombia \\ Jhonnatan Patiño \\ Residente de Medicina Interna de la Universidad de la \\ Sabana, Chía, Colombia \\ Alirio Bastidas \\ Médico internista, neumólogo, epidemiólogo, Clínica \\ Universidad de La Sabana, Chía, Colombia
}

a Correspondencia: martinarsanios@gmail.com

Cómo citar: Martín Arsanios DA, Quintero E, Benjumea Henao S, Arteaga A, Robles V, Vesga D, Patiño J, Bastidas A. Infarto agudo de miocardio y bloqueo de rama izquierda: utilidad de los criterios de Sgarbossa. Univ. Med. 2019;60(4). https:// doi.org/10.11144/Javeriana.umed60-4.sgar

\section{RESUMEN}

Introducción: En presencia de bloqueo de la rama izquierda, el infarto agudo de miocardio (IAM) es un reto diagnóstico para el médico. Objetivo: Evaluar la utilidad de los criterios de Sgarbossa en el diagnóstico de IAM en pacientes con bloqueo de la rama izquierda mediante una revisión sistemática y metaanálisis. Método: Búsqueda estructurada de la literatura en Medline, Lilacs, Ovid y Embase, desde enero 1996 hasta enero de 2018. Basados en los criterios de inclusión y exclusión, tres revisores seleccionaron los artículos que respondieran la pregunta de investigación. La calidad de los artículos se evaluó con el instrumento QUADAS-2. Resultados: Catorce estudios evaluaron los criterios de Sgarbossa utilizando un puntaje mayor o igual a 3 como corte. En total, fueron 3689 pacientes. La sensibilidad fue de 0,27 (IC95\%: 0,24-0,29; p = 0,0000); especificidad de 0,97 (IC95\%: 0,96-0,98; $\mathrm{p}=0,0001) \operatorname{LR}(+)$ de $10,95(6,28-19,11 ; \mathrm{p}=0,03)$ y $\operatorname{LR}(-)$ de 0,67 (0,56-0,81; $\mathrm{p}=0,0000)$. 
Conclusiones: Los criterios de Sgarbossa tienen una alta especificidad para el diagnóstico de IAM con bloqueo de la rama izquierda; pero bajo LR(-) y sensibilidad. Se deben evaluar nuevos algoritmos diagnósticos y validar para la población colombiana.

Palabras clave

infarto agudo de miocardio; síndrome coronario agudo; bloqueo de rama; diagnóstico; Colombia.

\begin{abstract}
Introduction: Acute myocardial infarction (AMI) in the presence of left bundle branch block (LBBB) is a diagnostic challenge for the physician. The purpose of this article is to evaluate the usefulness of the Sgarbossa criteria for the diagnosis of AMI in patients with LBBB through a systematic review and meta-analysis. Method: Structured searching of the literature in Medline, Lilacs, Ovid, and Embase from January 1996 to January 2018. Using the inclusion and exclusion criteria 3 reviewers selected the articles that answered the research question. The quality of the articles was evaluated using the QUADAS-2 tool. Results: 14 studies evaluated the Sgarbossa criteria using a score greater than or equal to 3. With a total of 3689 patients, the sensitivity was 0.27 (95\% CI: 0.24-0.29; p $=0.0000)$; specificity of 0.97 (95\% CI: 0.96-0.98; $\mathrm{p}=$ $0.0001) \operatorname{LR}(+)$ was $10.95(6.28-19.11 ; \mathrm{p}=0.03)$, and LR(-) was $0.67(0.56-0.81 ; p=0.0000)$. Conclusions: The Sgarbossa criteria have a high specificity for the diagnosis of AMI with LBBB; however, the sensitivity and the LR(-) are low. It is necessary to evaluate new diagnostic algorithms and to validate the criteria for Colombia.

Keywords

acute myocardial infarction; acute coronary syndrome; bundle branch block; diagnosis; Colombia.
\end{abstract}

\section{Introducción}

La presencia concomitante de bloqueo de la rama izquierda (BRI) y sospecha de infarto agudo de miocardio (IAM) supone un reto diagnóstico y terapéutico para el clínico (1). Por esta razón, surgen los criterios de Sgarbossa como herramienta que facilita el abordaje de estos pacientes (tabla 1) (2). Sin embargo, a pesar de tener una buena especificidad, en el estudio original, publicado en 1996, la sensibilidad apenas alcanza un 36\% (2). Esto se traduce en que un gran porcentaje de pacientes podrían quedar excluidos de las conductas de reperfusión por no tener el diagnóstico de IAM si no se logran interpretar adecuadamente estos criterios. Con el fin de evaluar si los criterios de Sgarbossa tienen un buen rendimiento para el diagnóstico de IAM en pacientes con BRI realizamos una revisión sistemática y metaanálisis.

\section{Tabla 1}

Criterios de Sgarbossa

\begin{tabular}{|l|c|}
\hline \multicolumn{1}{|c|}{ Criterios de Sgarbossa } & Puntos \\
\hline $\begin{array}{l}\text { Elevación concordante del segmento ST } \\
\text { mayor o igual a } 1 \mathrm{~mm} \text { en cualquier } \\
\text { derivación }\end{array}$ & 5 \\
\hline $\begin{array}{l}\text { Depresión del segmento ST mayor o } \\
\text { igual a } 1 \mathrm{~mm} \text { en V1, V2 o V3 }\end{array}$ & 3 \\
\hline $\begin{array}{l}\text { Elevación discordante del segmento ST } \\
\text { mayor o igual a } 5 \mathrm{~mm}\end{array}$ & 2 \\
\hline
\end{tabular}

Nota:primero, dos puntos son de concordancia; tercer punto, discordancia.

Más de 3 puntos se considera tiene

alta especificidad para el diagnóstico.

\section{Metodología}

Se realizó una revisión sistemática en las bases de datos Pubmed, Embase, OVID y Lilacs. La estrategia de búsqueda se realizó utilizando los términos: (acute myocardial infarction) OR (myocardial infarction) OR (coronary syndrome) AND (bundle branch block). Se tuvieron en cuenta artículos publicados desde el 1 de enero de 1996 hasta el 1 de enero de 2018, entre estos casos y controles y estudios de cohorte en inglés, español, portugués, francés e italiano. Se incluyeron pacientes adultos que se hubieron encontrado en urgencias, hospitalización o unidad de cuidado intensivo, con sospecha de IAM y presencia concomitante de BRI, en quienes se hubiera evaluado el rendimiento de un puntaje mayor o igual a 3 puntos en los criterios de Sgarbossa, en comparación con el patrón de referencia (gold standard). En cuanto a este último, dada la heterogeneidad en la definición del estándar y los escasos artículos, se tomó cualquiera de los siguientes: acuerdo interobservador, enzimas cardiacas positivas y cateterismo cardiaco positivo concordante con lesión arterial responsable de IAM. Se excluyeron aquellos estudios en los cuales se evaluó cada criterio por separado, sin incluir la evaluación de un puntaje mayor o igual a 3; adicionalmente, las embarazadas y la población pediátrica fue excluida de la revisión y metaanálisis. No se 
consideraron revisiones de tema, presentaciones de caso o series de caso.

Dos miembros del grupo investigador (DAMA y DVM) revisaron en las distintas bases de datos con todos los títulos y resúmenes de la búsqueda inicial. Ellos incluyeron y excluyeron los artículos para evaluación y, después, el resto del grupo investigador los revisó. Los datos se trasladaron a una hoja electrónica de Excel $^{\circledR}$, donde se evaluaron las características de los estudios y sus estimativos puntuales o medidas de resumen.

Para la evaluación de la calidad metodológica de los estudios incluidos y la calidad de la evidencia se utilizó el instrumento QUADAS-2, que es la herramienta actual recomendada para evaluar el riesgo de sesgos y la aplicabilidad de los estudios primarios de precisión diagnóstica que son utilizados en revisiones sistemáticas.

Mediante el uso de esta herramienta se evaluaron dos aspectos fundamentales: la proporción de preocupación que la aplicabilidad de los estudios primarios utilizados para la revisión sistemática genera y la proporción de preocupación que generan los estudios primarios en cuanto al riesgo de sesgos, esto por medio de la evaluación de flujo y tiempos, prueba de referencia, prueba de índice y selección de pacientes.

\section{Resultados}

En la búsqueda inicial se identificaron 3514 artículos, de los cuales se seleccionaron 17 para la revisión sistemática, 6 artículos se escogieron con la estrategia de "bola de nieve" basados en las referencias de distintos artículos de la búsqueda inicial, 12 correspondían a casos y controles y el restante a estudios de cohorte. De los 17 revisados, se optó por 15 para realizar el metaanálisis. Dos de ellos no aportaban datos para obtener la sensibilidad y especificidad de un puntaje mayor o igual a 3 en los criterios de Sgarbossa (figura 1).

\section{Figura 1}

Flujograma de búsqueda

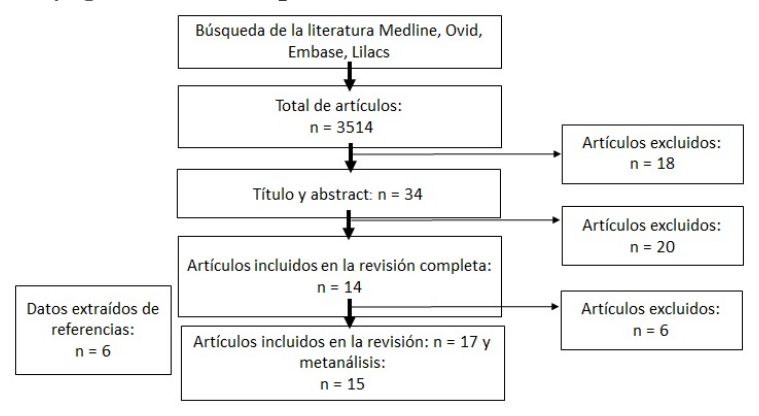

A diferencia del metaanálisis publicado en 2008 por Tabas et al. (3), se incluyó el artículo original de Sgarbossa (2), y no se excluyeron artículos que utilizaran los pacientes de este estudio. En los estudios incluidos, el IAM se determinó mediante enzimas cardiacas en algunos o todos los pacientes, acuerdo interobservador y uso de cateterismo cardiaco o la combinación de estas (tabla 2). Para un puntaje $\geq 3$ en los criterios de Sgarbossa obtuvimos una sensibilidad global de 0,27 (IC95\%: 0,24-0,29; $\mathrm{p}=0,0000$ ) (figura 2); una especificidad global de 0,97 (IC95\%: 0,96-0,98; p = 0,0001) (figura 3); un área bajo la curva de 0,90; likelihood ratio positivo $(\mathrm{LR}+)$ global de 10,95 (6,28-19,11; p = $0,03)$ y likelihood ratio negativo (LR-) global de $0,67(0,56-0,81 ; \mathrm{p}=0,0000)$ (figuras 4, 5, 6), todo esto mostrando gran heterogeneidad entre los diferentes estudios. 
Tabla 2

Comparación cualitativa de los estudios evaluados

\begin{tabular}{|c|c|c|c|c|}
\hline Estudios & \begin{tabular}{|c}
$\begin{array}{c}\text { Número de pacientes } \\
\text { (IAM + BRI) }\end{array}$ \\
\end{tabular} & \begin{tabular}{|c|} 
Acuerdo \\
interobservador
\end{tabular} & $\begin{array}{l}\text { Enzimas } \\
\text { cardiacas }\end{array}$ & \begin{tabular}{|c}
$\begin{array}{c}\text { Cateterismo } \\
\text { cardiaco }\end{array}$ \\
\end{tabular} \\
\hline $\begin{array}{l}\text { Jain et al. (2011) } \\
(5)\end{array}$ & $892(36)$ & 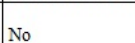 & No & 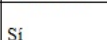 \\
\hline $\begin{array}{l}\text { Siu Fai Li et al. } \\
(2000)(19)\end{array}$ & $190(25)$ & Si & Si & No \\
\hline $\begin{array}{l}\text { Dod et al. (2016) } \\
\text { (4) }\end{array}$ & $162(33)$ & Si & $\mathrm{Si}$ & Si \\
\hline Gregg et al. (2013) & $239(141)$ & No & No & No \\
\hline $\begin{array}{l}\text { Di Marco et al. } \\
(2017)(20)\end{array}$ & $145(54)$ & Si & Si & Si \\
\hline $\begin{array}{l}\text { Maloy et al. }(2009) \\
(21)\end{array}$ & $156(57)$ & No & $\mathrm{Si}$ & No \\
\hline \begin{tabular}{|l}
$\begin{array}{l}\text { Smith et al. }(2012) \\
(12)\end{array}$ \\
\end{tabular} & $162(33)$ & Si & $\mathrm{Si}$ & Si \\
\hline \begin{tabular}{|l}
$\begin{array}{l}\text { Gula et al. (2003) } \\
(18)\end{array}$ \\
\end{tabular} & 499 (414) & No & No & No \\
\hline \begin{tabular}{|l} 
Kontos et al. \\
$(2000)(6)$
\end{tabular} & $182(24)$ & Si & Si & No \\
\hline $\begin{array}{l}\text { Gunnarsson et al. } \\
(2001)(22)\end{array}$ & $158(76)$ & Si & $\mathrm{Si}$ & No \\
\hline $\begin{array}{l}\text { Sgarbossa (muestra } \\
\text { de derivación) } \\
(1996)(2)\end{array}$ & $145(131)$ & Si & Si & No \\
\hline $\begin{array}{l}\text { Shlipak et al. } \\
(1999)(7)\end{array}$ & $89(31)$ & No & Si & No \\
\hline $\begin{array}{l}\text { Edhouse et al. } \\
\text { (1999) (8) }\end{array}$ & $50(26)$ & Si & $\mathrm{Si}$ & No \\
\hline $\begin{array}{l}\begin{array}{l}\text { Eriksson et al. } \\
(1999)(9)\end{array} \\
\end{array}$ & $33(14)$ & No & $\mathrm{Si}$ & No \\
\hline $\begin{array}{l}\text { Maynard et al. } \\
(2003)(23)\end{array}$ & $56(18)$ & No & Si & No \\
\hline $\begin{array}{l}\text { Wong et al. (2005) } \\
\text { (13) }\end{array}$ & $300(242)$ & No & Si & No \\
\hline $\begin{array}{l}\text { Meyers et al. } \\
(2015)(11)\end{array}$ & $294(45)$ & & $\mathrm{Si}$ & \\
\hline
\end{tabular}

Figura 2

Sensibilidad

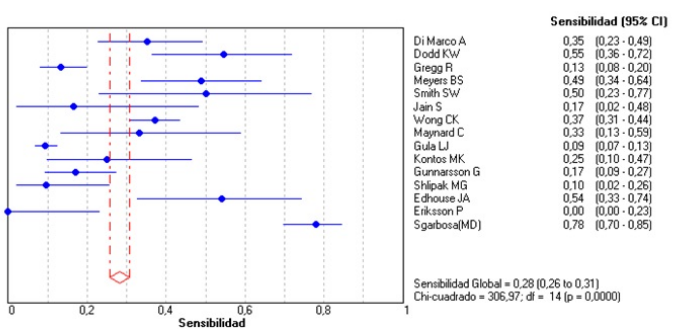

Figura 3

Especificidad

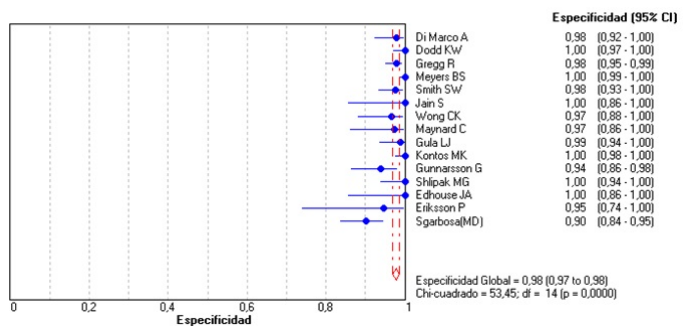

Figura 4

Likelihood ratio positivo

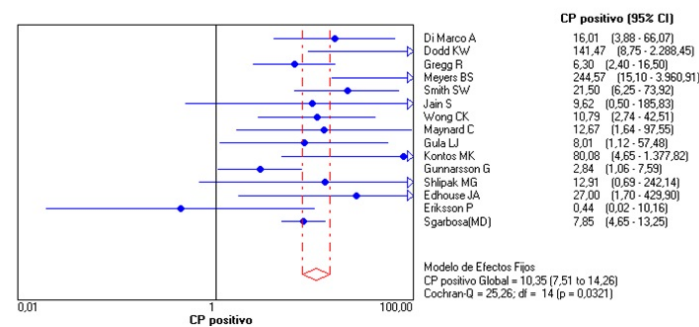

Figura 5

Likelihood ratio negativo
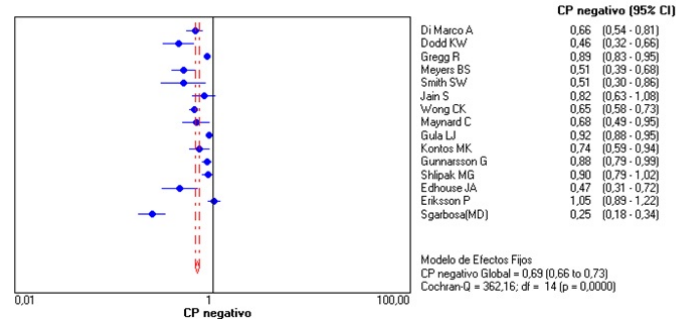

Figura 6

Área bajo la curva

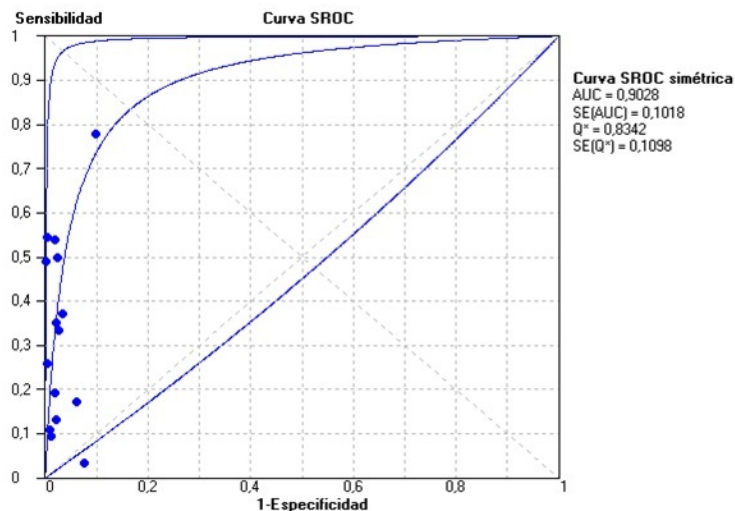

En 6 artículos $(4,5,6,7,8,9)$ no se logró determinar la $\mathrm{LR}+$, dado que su especificidad fue del 100\%, el 50\% de estos artículos no superaba los 100 pacientes y solo uno superaba los 500 pacientes (5), y en este último solo 36 pacientes tenían IAM + BRI. El número reducido de pacientes fue una dificultad de nuestro metaanálisis. A pesar de esto, los resultados encontrados son similares sin importar el tamaño de la muestra, como se puede observar en la tabla 3, y en las figuras 2 y 3 , correspondientes a sensibilidad y especificidad, un puntaje mayor o 
igual de 3 tiene una sensibilidad muy baja y una especificidad excelente en todos los artículos del metaanálisis.

\section{Tabla 3}

Resúmenes estimativos puntuales: evaluación de los parámetros estadísticos de los diferentes trabajos evaluados

\begin{tabular}{|c|c|c|c|c|}
\hline Autores & Sensibilidad (IC 95\%) & Especificidad (IC 95\%) & LR+ & LR- \\
\hline $\begin{array}{l}\text { Di Marco } \\
\text { et al. } \\
\text { (2017) }\end{array}$ & $35,19 \%(22,68-49,38 \%$ & $\begin{array}{r}97,80 \%(92,29 \%- \\
99,73 \%)\end{array}$ & $16,01(3,88-66,07)$ & $0,66(0,54-0,81)$ \\
\hline $\begin{array}{l}\text { Dodd et al. } \\
\text { (2016) }\end{array}$ & $\begin{array}{r}54,55 \%(36,35 \%- \\
71,89 \%)\end{array}$ & $\begin{array}{r}100,00 \%(97,18 \%- \\
100.0 \%) \\
\end{array}$ & $\mathrm{NA}$ & $0,45(0,31-0,66)$ \\
\hline $\begin{array}{l}\text { Gregg et } \\
\text { al. }(2013)\end{array}$ & $\begin{array}{r}13,29 \%(8,19 \%- \\
19,97 \%) \\
\end{array}$ & $\begin{array}{r}97,89 \%(95,15 \%- \\
99,31 \%) \\
\end{array}$ & $6,30(2,40-16,50)$ & $0,89(0,83-0,95)$ \\
\hline $\begin{array}{l}\text { Meyers et } \\
\text { al. (2015) }\end{array}$ & $\begin{array}{r}48,89 \%(33,70 \%- \\
64,23 \%)\end{array}$ & $\begin{array}{r}100,00 \%(98,53 \%- \\
100,00 \%)\end{array}$ & $\mathrm{NA}$ & $0,51(0,38-0,68)$ \\
\hline $\begin{array}{l}\text { Smith et al. } \\
\text { (2012) }\end{array}$ & $\begin{array}{r}50,00 \%(23,04 \%- \\
76,96 \%) \\
\end{array}$ & $\begin{array}{r}97,67 \%(93,35 \%- \\
99,52 \%) \\
\end{array}$ & $21,50(6,25-73,92)$ & $0,51(0,30-0,86)$ \\
\hline $\begin{array}{l}\text { Jain et al. } \\
\text { (2011) }\end{array}$ & $\begin{array}{r}16,67 \%(2,09 \%- \\
48,41 \%) \\
\end{array}$ & $\begin{array}{r}100,00 \%(85,75 \%- \\
100,00 \%) \\
\end{array}$ & $\mathrm{NA}$ & $0,83(0,65-1,07)$ \\
\hline $\begin{array}{l}\text { Wong et al. } \\
\text { (2005) }\end{array}$ & $\begin{array}{r}37,19 \%(31,08 \%- \\
43,61 \%)\end{array}$ & $\begin{array}{r}96,55 \%(88,09 \%- \\
99,58 \%)\end{array}$ & $10,79(2,74-42,51)$ & $0,65(0,58-0,73)$ \\
\hline $\begin{array}{l}\text { Maynard et } \\
\text { al. (2003) }\end{array}$ & $\begin{array}{r}33,33 \%(13,34 \%- \\
59,01 \%) \\
\end{array}$ & $\begin{array}{r}97,37 \%(86,19 \%- \\
99,93 \%) \\
\end{array}$ & $12,67(1,64-97,55)$ & $0,68(0,49-0,95)$ \\
\hline $\begin{array}{l}\text { Gula et al. } \\
\text { (2003) }\end{array}$ & $9,42 \%(6,78 \%-12,65 \%$ & $\begin{array}{r}98,82 \%(93,62 \%- \\
99,97 \%) \\
\end{array}$ & $8,01(1,12-57,49)$ & $0,92(0,88-0,95)$ \\
\hline $\begin{array}{l}\text { Kontos et } \\
\text { al. (2001) }\end{array}$ & $\begin{array}{r}25,00 \%(9,77 \%- \\
46,71 \%) \\
\end{array}$ & $\begin{array}{r}100,00 \%(97,62 \%- \\
100.0 \%)\end{array}$ & $\mathrm{NA}$ & $0,75(0,60-0,94)$ \\
\hline $\begin{array}{l}\text { Gunnarsson } \\
\text { et al. } \\
\text { (2001) }\end{array}$ & $\begin{array}{r}17,11 \%(9,43 \%- \\
27,47 \%)\end{array}$ & $\begin{array}{r}93,98 \%(86,50 \%- \\
98,02 \%)\end{array}$ & $2,84(1,06-7,59)$ & $0,88(0,79-0,99)$ \\
\hline $\begin{array}{l}\text { Shlipak et } \\
\text { al. (1999) }\end{array}$ & $\begin{array}{r}9,68 \%(2,04 \%- \\
25,75 \%)\end{array}$ & $\begin{array}{r}100,00 \%(93,84 \%- \\
100,00 \%) \\
\end{array}$ & $\mathrm{NA}$ & $0,90(0,80-1,01)$ \\
\hline $\begin{array}{l}\text { Edhouse et } \\
\text { al. (1999) }\end{array}$ & $\begin{array}{r}54,17 \%(32,82 \%- \\
74,45 \%) \\
\end{array}$ & $\begin{array}{r}100,00 \%(85,75 \%- \\
100,00 \%) \\
\end{array}$ & $\mathrm{NA}$ & $0,460,30-0,71$ \\
\hline $\begin{array}{l}\text { E. Eriksson et } \\
\text { al. (1999) }\end{array}$ & $0,00 \%(0,00 \%-23,16 \%$ & $\begin{array}{r}94,74 \%(73,97 \%- \\
99,87 \%)\end{array}$ & $\mathrm{NA}$ & $1,06(0,95-1,17)$ \\
\hline $\begin{array}{l}\text { Sgarbossa } \\
\text { (muestra de } \\
\text { derivación) } \\
(1996)\end{array}$ & $\begin{array}{r}77,86 \%(69,78 \%- \\
84,65 \%)\end{array}$ & $\begin{array}{r}90,08 \%(83,63 \%- \\
94,61 \%)\end{array}$ & $7,85(4,65-13,25)$ & $0,25(0,18-0,34)$ \\
\hline
\end{tabular}

NA:no aplica.

En cuanto a la definición de IAM, hay una amplia heterogeneidad en cuanto al patrón de referencia para definir infarto: a menos del 10\% de los pacientes se les realizó cateterismo cardiaco y solo 5 estudios $(4,5,10,11,12)$ se evaluaron con esta estrategia diagnóstica que correspondería al mejor método utilizado en todos los artículos. La calidad de la evidencia de cada artículo fue calificada con el instrumento QUADAS-2 (tablas 4 y 5 ; figuras 7 y 8 ).

\section{Tabla 4}

Instrumento QUADAS2 para evaluar la calidad de los estudios de precisión diagnóstica

\begin{tabular}{|l|l|l|l|l|}
\hline \multicolumn{1}{|c|}{ Autores } & Selección de pacientes & Prueba indice & Prueba de referencia & Flujo y tiempos \\
\hline Di Marco et al. & Bajo & Bajo & Alto & Bajo \\
\hline Dodd et al. & Bajo & Bajo & Alto & Bajo \\
\hline Gregg et al. & No claro & Bajo & Alto & No claro \\
\hline Meyers et al. & Bajo & Bajo & Bajo & Bajo \\
\hline Smith et al. & Bajo & Bajo & Bajo & Bajo \\
\hline Jain et al. & Bajo & Alto & Bajo & Bajo \\
\hline Wong et al. & Bajo & Bajo & Bajo & Bajo \\
\hline Maynard et al. & Bajo & Bajo & Alto & No claro \\
\hline Gula et al. & No claro & Alto & Alto & Alto \\
\hline Konto et al. & Bajo & Alto & Bajo & No claro \\
\hline Gunnarsson et al. & Bajo & Bajo & Alto & Bajo \\
\hline Shlipak et al. & Bajo & Bajo & Bajo & Bajo \\
\hline Edhouse et al. & Bajo & Alto & Bajo & No claro \\
\hline Eriksson et al. & Bajo & Bajo & Alto & Alto \\
\hline Sgarbossa & Alto & Bajo & No claro & Alto \\
\hline & & & & \\
\hline
\end{tabular}

Tabla 5

Instrumento QUADAS2 para evaluar la calidad de los estudios de precisión diagnóstica

\begin{tabular}{|c|c|c|c|c|c|c|c|}
\hline \multirow[t]{2}{*}{ Estudio } & \multicolumn{4}{|c|}{ Probabilidad de sesgos } & \multicolumn{3}{|c|}{$\begin{array}{c}\text { Preocupación sobre la } \\
\text { aplicabilidad de los resultados }\end{array}$} \\
\hline & \begin{tabular}{|c|}
$\begin{array}{c}\text { Selección } \\
\text { de los } \\
\text { individuos }\end{array}$ \\
\end{tabular} & \begin{tabular}{|c|} 
Prueba \\
indice
\end{tabular} & $\begin{array}{c}\text { Prueba } \\
\text { de } \\
\text { referencia }\end{array}$ & \begin{tabular}{|l|} 
Flujo y \\
tiempos
\end{tabular} & \begin{tabular}{|c|}
$\begin{array}{c}\text { Selección } \\
\text { de } \\
\text { pacientes }\end{array}$ \\
\end{tabular} & $\begin{array}{c}\text { Prueba } \\
\text { índice }\end{array}$ & $\begin{array}{c}\begin{array}{c}\text { Prueba } \\
\text { de } \\
\text { referencia }\end{array} \\
\end{array}$ \\
\hline $\begin{array}{l}\text { Di Marco } \\
\text { et al. }\end{array}$ & (ㄷ) & (2) & () & () & (9) & (2) & (2) \\
\hline $\begin{array}{l}\text { Dodd et } \\
\text { al. }\end{array}$ & (ㄹ) & (2) & () & () & ()ㅜㅇ & () & (e) \\
\hline $\begin{array}{l}\text { Gregg et } \\
\text { al. }\end{array}$ & ? & (ㄷ) & (2) & ? & (2) & (1) & (ㄷ) \\
\hline $\begin{array}{l}\text { Meyers et } \\
\text { al. }\end{array}$ & (ㄷ) & () & ()ㅜㅇ & () & () & (ㄷ) & () \\
\hline $\begin{array}{l}\text { Smith et } \\
\text { al. }\end{array}$ & () & () & () & () & ()ㅜㅇ & () & ()ㅜㅇ \\
\hline Jains et al. & (ㄷ) & ()ㅜㅇ & (ㄷ) & () & (ㄱ) & (i) & (ㄷ) \\
\hline $\begin{array}{l}\text { Wong et } \\
\text { al. }\end{array}$ & (ㅇ) & (웅 & () & (ㄱ) & () & () & () \\
\hline $\begin{array}{l}\text { Maynard } \\
\text { et al. }\end{array}$ & (ㄷ) & () & () & $?$ & ()ㅜㅇ & () & () \\
\hline Gula et al. & $?$ & (2) & (6) & (2) & (ㄷ) & (ㄷ) & () \\
\hline $\begin{array}{l}\text { Konto et } \\
\text { al. }\end{array}$ & (ㄷ) & ()ㅜㅇ & (ㄷ) & ? & () & ()ㅜㅇ & (ㄱ) \\
\hline $\begin{array}{l}\text { Gunnarson } \\
\text { et al. }\end{array}$ & (ㄷ) & ()ㅜ & (2) & () & () & () & (2) \\
\hline $\begin{array}{l}\text { Shlipak et } \\
\text { al. }\end{array}$ & (ㄷ) & (ㄷ) & ()ㅜㅇ & () & (ㄷ) & (2) & ()ㅜㅇ \\
\hline $\begin{array}{l}\text { Edhouse } \\
\text { et al. }\end{array}$ & (ㄷ) & (2) & ()ㅜㅇ & ? & ()ㅜ & (2) & () \\
\hline $\begin{array}{l}\text { Eriksson } \\
\text { et al. }\end{array}$ & () & ()ㅜㅇ & (2) & ()ㅜㅇ & () & () & (2) \\
\hline Sgarbossa & ()ㅜㅇ & ? & $?$ & ()ㅜㅇ & (ㄷ) & ()ㅜㅇ & (2) \\
\hline
\end{tabular}

\section{Figura 7}

QUADAS-2: riesgo de sesgo

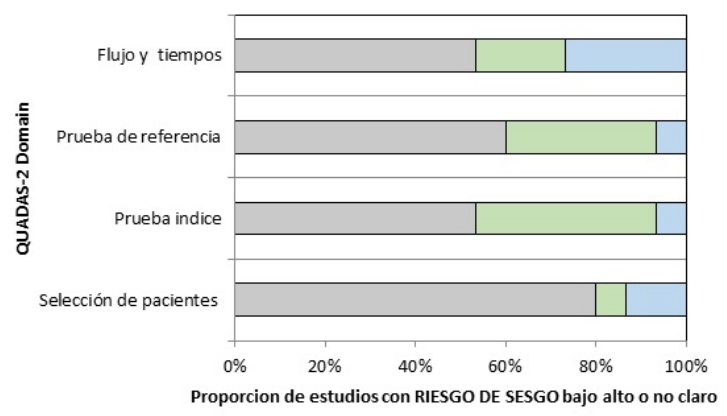

Figura 8

QUADAS-2: aplicabilidad

口Low $\square$ High $\square$ Unclear

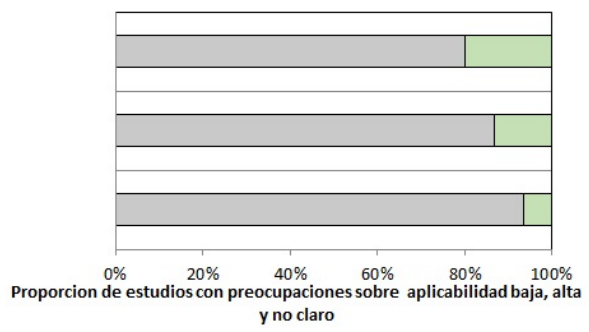




\section{Discusión}

El diagnóstico del IAM en pacientes con BRI es un reto diagnóstico para el clínico. Se estima una prevalencia de BRI en pacientes con IAM de entre el $2 \%$ y el $9 \%(13,14)$. En el estudio Assessment of Safety an Efficacy of a New Thrombolytic 2 y 3, una elevación importante de la creatina fosfocinasa-MB se presentó en el $62,5 \%$ de los 253 pacientes que cursaban con BRI $(14,15)$. La prevalencia de BRI en pacientes con IAM varía considerablemente y más aún en aquellos estudios donde se utilizaron enzimas como método de referencia para definir IAM. En aquellos estudios en los cuales se usa el cateterismo cardiaco, la prevalencia es más uniforme y un poco mayor en comparación con aquellos que usan las enzimas cardiacas, que está entre el 15\% y el 35\% (5,14). Por esta razón, desde hace más de una década, distintos investigadores han intentado desarrollar algoritmos diagnósticos para aumentar la detección o tener una forma más adecuada para detectar e identificar pacientes con IAM que cursen con BRI. Los criterios de Sgarbossa (tabla 1) son los más conocidos; sin embargo, a pesar de ser usados frecuentemente en la práctica clínica, su sensibilidad y especificidad varían entre estudios y el conocimiento de su utilidad y aplicación es poco clara.

Basados en esta premisa, nuestro metaanálisis evaluó la eficacia del uso de los criterios de Sgarbossa. Con los resultados globales del rendimiento de estos criterios para la predicción de IAM asociado a BRI con un puntaje $\geq 3$, observamos que, debido a su baja sensibilidad, no se puede descartar la presencia de IAM cuando este puntaje no es superior a lo indicado; por el contrario, dada su alta especificidad (figura 3), valor predictivo positivo y LR+ (figura 4) nos permite diagnosticar con IAM a aquellos pacientes que cumplan los criterios. No obstante, cabe recalcar que la decisión de la conducta del médico siempre debe tomarse de acuerdo con la presentación clínica del paciente. La mayoría de estos estudios reportados no evalúan las distintas escalas pronósticas que involucran el examen físico, los factores de riesgo, las enzimas cardiacas y el electrocardiograma en conjunto. Así es como las guías europeas de 2017 sobre el IAM con elevación del ST afirman que estas ayudas diagnósticas sirven "para asistir el diagnóstico" y la sospecha clínica de isquemia miocárdica en conjunto con todos estos hallazgos debe ser lo que defina el manejo (16).

De acuerdo con el metaanálisis realizado por Tabas et al. (3), en 2008, la sensibilidad que se obtuvo cuando el puntaje de los criterios de Sgarbossa era mayor o igual a 3 correspondió a un 20\%; mientras que la especificidad fue del $98 \%$ y tuvo un LR+ de 7,9, que resultaba clínicamente útil, pues en ese momento se correlacionaba con 7 de los 10 criterios electrocardiográficos usados para diagnosticar IAM en pacientes sin bloqueo de rama. También midieron las mismas variables cuando el puntaje era mayor o igual a 2 y se encontró que tanto la sensibilidad como la especificidad no mejoraban y, en cambio, se volvían heterogéneas, por lo que no resultaba de utilidad clínica (3).

Cabe anotar que en esa investigación no se incluyó el artículo original de Sgarbossa. Por su parte, en el presente estudio, basándose en puntajes mayores o iguales a 3, la sensibilidad que se alcanzó de manera global fue del $27 \%$, que continúa siendo baja a pesar del pequeño incremento (la especificidad fue del 97\%), lo cual concuerda con los resultados del metaanálisis anterior. No obstante, a pesar de esta concordancia con los estudios y el anterior metaanálisis, cabe mencionar algunas limitaciones encontradas. En primer lugar, la muestra de pacientes con IAM y BRI sigue siendo pequeña para llegar a obtener unos intervalos de confianza más adecuados. Por otra parte, la definición de IAM en los estudios encontrados es muy variable y no representan lo usado actualmente; los estudios publicados antes de 2008 en su mayoría utilizaban creatina cinasa como estrategia para el diagnóstico de IAM; entre tanto, los estudios realizados desde 2010 aumentaron el uso de las troponinas. Sin embargo, estos $(4,5,10,11,12,17)$, aunque se acercan más a la actualidad, difieren en el uso de cateterismo cardiaco y, en ocasiones, en la 
definición del punto J y distintas variables a la hora de interpretar los algoritmos.

Algunos estudios recientes han planteado nuevos criterios basados en los de Sgarbossa, como es el caso del algoritmo de Smith, que incluye los criterios y puntajes de Sgarbossa; pero con una modificación en el tercer criterio, lo cual hace más proporcional la elevación discordante del segmento ST de $1 \mathrm{~mm}$. Con esta modificación se observó un aumento en la sensibilidad de los criterios de Sgarbossa como método diagnóstico basado en el electrocardiograma para diferenciar pacientes con BRI con un evento coronario agudo o sin este, sobre todo cuando estos presentan oclusión de la arteria descendente anterior $(10,12,18,19,20,21,22,23)$. No obstante, investigaciones más recientes han demostrado que los criterios de Sgarbossa incluidos en las reglas de Smith continúan presentando una sensibilidad subóptima $(24,25)$. Las reglas 1,2 y 3 de Smith mostraron una sensibilidad del 67\%, del 54 y del 28\%, respectivamente; mientras que la especificidad continuó siendo considerable, por encima del 90\% en las tres.

\section{Conclusión}

Los criterios de Sgarbossa tienen una alta especificidad para el diagnóstico de IAM + BRI; sin embargo, poseen una baja sensibilidad y CP negativos muy bajos. Por lo anterior, estos criterios se pueden usar como una herramienta para el diagnóstico asociado a la clínica del paciente, que debe primar en caso de sospechar un síndrome coronario agudo. Se deben validar y estudiar estos criterios en la población colombiana, para tener un concepto del rendimiento de esta estrategia en nuestros pacientes.

\section{Referencias}

1.Sgarbossa EB. Value of the ECG in suspected acute myocardial infarction with left bundle branch block. J Electrocardiol. 2000 Jan;33:87-92.
2.Sgarbossa EB, Pinski SL, Barbagelata A, Underwood DA, Gates KB, Topol EJ, et al. Electrocardiographic diagnosis of evolving acute myocardial infarction in the presence of left bundle-branch block. N Engl J Med. 1996 Feb 22;334(8):481-7.

3.Tabas JA, Rodríguez RM, Seligman HK, Goldschlager NF. Electrocardiographic criteria for detecting acute myocardial infarction in patients with left bundle branch block: A meta-analysis. Ann Emerg Med. 2008;52(4):329-36. https:// doi.org/10,1016/

j.annemergmed.2007,12.006

4.Dodd KW, Elm KD, Smith SW. Comparison of the QRS complex, STsegment, and T-wave among patients with left bundle branch block with and without acute myocardial infarction. J Emerg Med. 2016;51(1):1-8.

5.Jain S, Ting HT, Bell M, Bjerke CM, Lennon RJ, Gersh BJ, et al. Utility of left bundle branch block as a diagnostic criterion for acute myocardial infarction. Am J Cardiol. 2011 Apr 15;107 (8):1111-6.

6.Kontos MC, McQueen RH, Jesse RL, Tatum JL, Ornato JP. Can myocardial infarction be rapidly identified in emergency department patients who have left bundle-branch block? Ann Emerg Med. 2001 May;37(5):431-8.

7.Shlipak MG, Lyons WL, Go AS, Chou TM, Evans GT, Browner WS. Should the electrocardiogram be used to guide therapy for patients with left bundle-branch block and suspected myocardial infarction? JAMA. 1999 Feb;281(8):714.

8.Edhouse JA, Sakr M, Angus J, Morris FP. Suspected myocardial infarction and left bundle branch block: electrocardiographic indicators of acute ischaemia. J Accid Emerg Med. 1999;16(5):331-5. 
9.Eriksson P, Gunnarsson G, Dellborg M. Diagnosis of acute myocardial infarction in patients with chronic left bundle-branch block. Standard 12-lead ECG compared to dynamic vectorcardiography. Scand Cardiovasc J. 1999;33(1):17-22.

10.Di Marco A, Anguera I, Rodríguez M, Sionis A, Bayes-Genis A, Rodríguez $\mathrm{J}$, et al. Assessment of smith algorithms for the diagnosis of acute myocardial infarction in the presence of left bundle branch block. Rev Esp Cardiol. 2017 Jul;70(7):559-66.

11.Meyers HP, Limkakeng AT, Jaffa EJ, Patel A, Theiling BJ, Rezaie SR, et al. Validation of the modified Sgarbossa criteria for acute coronary occlusion in the setting of left bundle branch block: A retrospective case-control study. Am Heart J. 2015 Dec;170(6):1255-64.

12.Smith SW, Dodd KW, Henry TD, Dvorak DM, Pearce LA. Diagnosis of ST-elevation myocardial infarction in the presence of left bundle branch block with the ST-elevation to S-wave ratio in a modified Sgarbossa rule. Ann Emerg Med. 2012 Dec;60(6):766-76.

13.Wong CK, French JK, Aylward PEG, Stewart RAH, Gao W, Armstrong $\mathrm{PW}$, et al. Patients with prolonged ischemic chest pain and presumednew left bundle branch block have heterogeneous outcomes depending on the presence of ST-segment changes. J Am Coll Cardiol. 2005;46(1):29-38.

14.Mehta N, Huang HD, Bandeali S, Wilson JM, Birnbaum Y. Prevalence of acute myocardial infarction in patients with presumably new left bundlebranch block. J Electrocardiol. 2012 Jul;45(4):361-7.

15.Al-Faleh H, Fu Y, Wagner G, Goodman S, Sgarbossa E, Granger $\mathrm{C}$, et al. Unraveling the spectrum of left bundle branch block in acute myocardial infarction: Insights from the Assessment of the Safety and Efficacy of a New Thrombolytic (ASSENT 2 and 3) trials. Am Heart J. 2006 Jan;151(1):10-5.

16.Ibanez B, James S, Agewall S, Antunes MJ, Bucciarelli-Ducci C, Bueno H, et al. 2017 ESC guidelines for the management of acute myocardial infarction in patients presenting with ST-segment elevation. Eur Heart J. 2018 Jan;39(2):119-77.

17.Gregg RE, Helfenbein ED, Babaeizadeh S. New ST-segment elevation myocardial infarction criteria for left bundle branch block based on QRS area. J Electrocardiol. 2013;46(6):528-34.

18.Gula LJ, Dick A, Massel D. Diagnosing acute myocardial infarction in the setting of left bundle branch block: prevalence and observer variability from a large community study. Coron Artery Dis. 2003 Aug;14(5):387-93.

19.Li SF, Walden PL, Marcilla O, Gallagher EJ. Electrocardiographic diagnosis of myocardial infarction in patients with left bundle branch block. Ann Emerg Med. 2000;36(6):561-5.

20.Sionis A, Bayes-genis A, Marco A Di, Anguera I, Rodrı M, Ariza-sole A, et al. Assessment of smith algorithms for the diagnosis of acute myocardial infarction in the presence of left bundle branch block. 2016;70 (7):559-66.

21.Maloy KR, Bhat R, Davis J, Reed K, Morrissey R. Sgarbossa criteria are highly specific for acute myocardial infarction with pacemakers. West J Emerg Med. 2010;11(4):354-7.

22.Gunnarsson G, Eriksson P, Dellborg M. ECG criteria in diagnosis of acute myocardial infarction in the presence of left bundle branch block. Int J Cardiol. 2001 Apr;78(2):167-74. 
23.Maynard SJ, Menown IB, Manoharan G, Allen J, Mc CAJ, Adgey AA. Body surface mapping improves early diagnosis of acute myocardial infarction in patients with chest pain and left bundle branch block. Heart. 2003;89(9):998-1002. 